\title{
L'ÉLEVAGE NATUREL DE L'ALEVIN DE TRUITE FARIO
}

\author{
par le Comte Victor D'ANSEMBOURG \\ Pisciculteur à Assenois (Luxembourg belge)
}

\section{1. - IL Y AVAIT DU BON DANS LES VIEILLES MÉthodES}

Aux pages consacrées par les ouvrages spéciaux à l'historique de la pisciculture, nous lisons que Remy et GÉHIN, deux modestes pêcheurs des Vosges, réinventant, en 1842 - il y a donc un siècle - le procédé de JAcoBy, pratiquèrent sur des oufs de Truite, la fécondation dite " artificielle " et réussirent à élever les alevins obtenus. On ne nous dit pas quel fut exactement le mode d'élevage expérimenté, mais, s'il est vrai que des milliers de Truites, âgées de un à trois ans, furent ainsi produites en étang, il est très improbable que des essais de nourrissage, du genre de ceux qui devaient donner de si cuisantes déceptions quelques années plus tard, aient pu constituer le fondement de ce beau résultat. Nous pensons donc que le traitement fut naturel dès la résorption et que l'on procéda au déversement en étang, sinon à ce moment précis, du moins à un âge peu avancé, puisque les récoltes comptaient des sujets d'un an. Cette tentative, qui appartient pour ainsi dire à la préhistoire de la science piscicole, est sans doute voisine de la méthode dont il sera question dans le présent article.

Vint ensuite, sous l'impulsion de Coste, la période de vulgarisation de cette nouvelle activité offcrte aux hommes. C'est vers 1853 que furent tentés, en Belgique, les premiers essais inspirés des procédés de ce fervent propagateur. Faut-il situer la date de naissance de la Trutticulture artificielle belge, autour de cette année, ou la faire remonter aux repeuplements ordonnés antérieurement par Léopold $I^{\text {er }}$, et effectués, nous dit-on, avec succès? Nous l'ignorons, car nous ne sommes pas en possession de renseignements suffisants sur les secrets de fabrication et sur la provenance (était-elle indigène ou étrangère ?) des alevins qui avaient servi à ces déversements " précurseurs".

A l'engouement des premiers jours, suivi de pas mal d'échecs, dus notamment à la vaine recherche d'un aliment factice, succéda une période de découragement et d'abandon, et, ce n'est qu'en 1884 que s'ouvrit franchement l'ère des repeuplements officiels et réguliers. Les pépinières de 
Salmonidés essaimèrent alors. Comme certaines de leurs aînées, qui avaient renoncé au nourrissage, elles se contentèrent, au début, de faire éclore mais, cette fois, en quantités innombrables - les œufs récoltés sur place, ou achetés au dehors, lâchant les alevins peu avant la résorption de leur vésicule, c'est-à-dire, dès qu'il devenait indispensable de leur procurer une nourriture, dont on n'avait pas encore trouvé le bon succédané. Les auteurs contemporains motivaient ce licenciement précoce des élèves par d'autres raisons encore, les unes discutables, les autres plus pertinentes. Plus tard, la " découverte de la rate ", réalisée en 1886 à l'Aquarium du Trocadéro, permit à quelques adeptes de ce procédé d'alimentation de prolonger la période de stabulation. Cependant les réfractaires étaient plus nombreux que ceux-ci, et, somme toute, la pratique du nourrissage ne se généralisa que 15 ou 20 années après son invention.

Cette époque connut certes des expériences malheureuses, mais elles sont bien moins imputables, nous semble-t-il, à des erreurs essentielles de traitement qu'à la manie de l'acclimatation qui sévissait alors et qui fut l'origine incontestable d'un grand gaspillage d'alevins. La Truite fario figurait, néanmoins, en nombres imposants, parmi les sujets d'espèces plus ou moins hétéroclites qui étaient introduits dans les eaux les plus diverses, et, l'on peut admettre que ces déversements atteignaient au moins partiellement leur but. Un sérieux accroissement de la consommation des poissons d'eau douce, en général, et de la Truite, en particulier, suivit de près ces premiers repeuplements effectués par l'Etat. Sans doute, la multiplication concomitante des pêcheurs, réguliers ou braconniers, coopéra à cette augmentation des captures, mais, celle-ci doit, croyons-nous, être inscrite, pour une bonne part, au crédit des déversements.

Il ne faut donc pas condamner en bloc les méthodes de nos grandsparents. Bien qu'obligés de marcher à tâtons sur un terrain neuf et dépourvus de traditions, ils ont disséminé de la bonne graine. Il nous appartient d'en récolter les fruits. Pratiquons les élagages que nous dictent nos expériences, jointes aux leurs, mais considérons notre propre savoir avec l'humilité que requiert, par exemple, sa comparaison avec la somme des acquisitions millénaires qui constituent le patrimoine scientifiqué de l'Agriculture, et puis, rendons un hommage équitable, reconnaissant même, aux travaux, ainsi qu'aux écrits désintéressés, de ces pionniers.

Si nous avons semé, dès le début d'un article, qui ne doit pas être une compilation (1), quelques bribes inspirées de la littérature piscicole du déclin du siècle dernier, c'est que ce bref retour sur le passé nous offre un

(1) Nous voudrions en effet limiter la présente étude aux " choses vues et éprouvées ». Celles-ci étant restreintes à deux zones géologiques du Luxembourg belge (l'Ardenne et le Jurassique), certaines conclusions qui en découleront, ne seront peut-être pas applicables à toutes les régions. Nous tâcherons donc de ne pas généraliser ce qui aurait un caractère par trop local. 
enseignement qui mérite d'être considéré comme le premier principe de l'élevage naturel de la Truite. En effet, si l'on cherche ce qu'il y avait de bon dans les vieux procédés, on peut trouver et retenir ceci : avant l'emploi de la rate, les alevins étaient lâchés dans leur prime jeunesse, munis encore d'un fragment de leur sac vitellin. Nous ne discuterons pas l'opportunité du déversement immédiat de ces bébés de poisson dans les eaux libres (on reconnaft actuellement que, si ceux-ci conviennent à tel endroit, les truitelles sont plus indiquées pour tel autre), mais ce que nous voulons souligner, c'est le fait que les laboratoires d'incubation et d'alevinage étaient évacués avant l'élevage proprement dit, et c'est là le premier principe dont nous parlions. Partout où la chose est possible, et, où l'on s'est écarté de cette bonne règle, c'est à cela qu'il convient de revenir, en ce qui concerne la Truite fario.

On constate, aujourd'hui, une certaine réaction contre l'alimentation artificielle des alevins de cette espèce; il y a même, en Belgique notamment, des établissements de Trutticulture qui sont restés fidèles à la méthode extensive; néanmoins cette tendance ne nous semble pas toujours assez radicale. Des auteurs modernes, par exemple, conseillent bien d'offrir à l'alevin des conditions de vie plus naturelles, mais ils s'obstinent à faire allusion à un nourrissage artificiel préalable, dont nous ne voyons pas la nécessité dans les circonstances habituelles.

\section{2. - Motifs qui Plaident en faveur de l'élevage naturel}

Précisons d'abord le sens donné ici à l'élevage naturel. Ces mots ne signifieront pas l'abandon intégral à la nature du cycle complet des opérations piscicoles, c'est-à-dire, le renoncement aux méthodes usuelles de la ponte, de la fécondation et de l'incubation, mais simplement la suppression absolue, pour la Truite fario, du nourrissage artificiel de l'alevin, jusqu'au moment où il devient Truitelle d'un été.

Nous laisserons aussi de côté les procédés de distribution de nourriture vivante dans les bacs d'alevinage. La récolte, ou la culture en bassins, de micro-Crustacés et de larvules, peuvent être recommandables dans certains cas isolés qui dictent cette solution intermédiaire, mais nous ne pensons pas qu'elles soient d'application courante, car il est à peu près impossible de produire ainsi, ou de recueillir, cette faune nutritive en quantités suffisantes et en temps opportun. Disons d'ailleurs, qu'il y a souvent moyen de faire mieux que cela, c'est-à-dire de mettre l'alevin en contact direct avec cette manne bienfaisante, disponible en bien plus grandes quantités, dans des étangs, chauds à la surface et frais au fond, où peuvent s'associer ces deux productions, peu compatibles à première vue : celle du zooplancton et celle de la Truite. 
Ce qu'il faut à l'alevin : Proies vivantes, Espace, Soleil.

Les raisons qui militent en faveur du traitement natured procèdent du souci de supprimer tout ce qui s'écarte inutilement des lois normales de la vie d'une espèce animale, particulièrement rebelle aux contraintes imposées à son caractère. Nous devons bien pratiquer sous le signe de l'artificiel, la ponte, la fécondation, I'incubation et la majeure partie de la résorption ; mais, n'est-ce point assez? Ce traitement qui violente la nature durant quatre opérations consécutives, se justifie par des exigences de rendement, de surveillance et de protection ; l'expérience nous enseigne que les alevins de certaines espèces le supportent étonnamment bien, pourvu qu'il soit appliqué suivant les règles de l'art; soit. Après cela cependant, vient la phase capitale de leur existence, celle où ils commencent à prélever, de plus en plus, aux éléments ambiạnts, les matériaux de leur propre substance, celle qui marque pour eux le début du développement, disons même le début de la vie réelle, en opposition avec la vie végétative qu'ils menaient dans l'œuf et pendant la période vitelline. Alors, si nous voulons leur donner toutes les chances de prendre un bon départ, et d'accomplir sans grand danger cette période si importante, il est temps de changer de méthode.

Ils étaient entassés par milliers dans des clos de quelques décimètres carrés. Offrons-leur dès à présent, l'espace, la liberté de mouvements, le bien-être favorable à leur prospérité, la nourriture vivante qui répond spécifiquement à leur appétit, et, enfin le soleil. Car remarquons-le bien, contrairement à une opinion trop répandue, la jeune Truite, dès qu'elle est débarrassée de sa vésicule et qu'elle cherche sa pitance, aime la lumière à ses heures. Il suffit, pour s'en convaincre, de voir, par une belle matinée d'Avril et de Mai, les alevins, même ceux de fario, se délecter à la surface de l'eau et en plein soleil. La recherche de la nourriture a-t-elle une part plus ou moins importante dans cet attrait? Y a-t-il uniquement héliotropisme? Il est possible que ces deux causes interviennent ici ; mais, quoi qu'il en soit, il est tout indiqué de donner à nos élèves cette possibilité de voir la lumière quand ils en auront besoin, au même titre qu'on leur procure l'ombre et les abris, qu'ils utiliseront à leur gré.

\section{Ecueils de l'élevage artificiel de la fario.}

Si les perfectionnements que l'on a apportés à la pisciculture, en instituant le nourrissage en stabulation du poisson encore vésiculé, ont trouvé un emploi intéressant dans le traitement de la Truite arc-en-ciel, il faut reconnaître que cette innovation n'a pas entièrement répondu aux besoins de la fario. Existe-t-il beaucoup de praticiens qui obtiennent, par la méthode intensive de l'alimentation purement artificielle, pratiquée, dès avant la résorption, sur les alevins de cette espèce, des résultats franchement satisfaisants? Certes, le savoir-faire, la ténacité des soins, une eau parti- 
culièrement propice, une ascendance adaptée à ces procédés, nous voyons là autant de facteurs qui peuvent aplanir partiellement les aspérités de cette méthode, néanmoins, on sera rarement récompensé de ses peines par des rendements numériques, - calculés de l'œuf à la truitelle d'automne - égaux à ceux que peut fournir la Truite arc-en-ciel saine et de bonne race, élevée de la même façon, ou la fario, livrée à elle-même, dans un étang d'élevage.

On sait que celle-ci s'accommode difficilement du nourrissage, pendant son stade juvénile. Elle possède - surtout si elle appartient à une race indigène, originaire des ruisseaux - une hérédité sauvage, farouche, qui se manifeste déjà dans les bacs d'éclosion par les grouillements continuels qui animent les quatre coins de l'appareil, dans lesquels les alevins tentent obstinément de se cacher. L'accoutumance à happer les particules nutritives que l'on répand dans l'eaư à la disposition de ceux-ci est désespérément lente à obtenir, et, en attendant, il y a des victimes de la faim. Cet apprentissage est particulièrement laborieux dans les eaux très froides, où l'on risque d'assister à des désastres, avant de réussir à apprivoiser les survivants affamés.

Tout cela entraîne en outre un surcroît de besogne compliquée, et, surtout, un risque permanent d'infection. Aussi, disons-le franchement, l'alimentation artificielle de l'alevin ne peut se justifier que dans des cas différents de ceux que nous envisageons : par exemple, - pour la Truite arcen-ciel, plus apprivoisée, plus accommodante, et parcourant d'habitude, avec une soumission parfaite aux heures de repas, les étapes qui la mènent à la taille d'une simple ou d'une double portion; - et pour la fario, quand le milieu destiné à recevoir l'alevin ne peut être prêt en même temps que celui-ci, quand les espaces nécessaires font défaut, bref lorsqu'il n'y a pas moyen de faire autrement.

Certains Trutticulteurs attachent une importance vitale au fait d'alimenter les poissons fraîchement éclos, non seulement quand ils commen. cent à s'agiter, manifestant ainsi leur besoin de nourriture extérieure, mais dès l'âge de quelques jours, de façon à bien s'assurer qu'ils ne manqueront de rien. A cette fin, on leur envoie d'épais nuages de rate diluée, on leur donne ainsi, plusieurs fois par jour, un véritable bain de jus de viande, et l'on se dit qu'ils en prendront bien quelques parcelles. Un tel empressement à salir les bacs doit être ralenti, mais il s'impose, souvent, jusqu'à un certain point, à cause des lenteurs de l'éducation alimentaire de l'alevin de fario, et, c'est là une aggravation des inconvénients, déjà signalés, de la méthode du nourrissage.

Dans l'élevage naturel, la période de stabulation est conduite de façon autrement simple et salutaire. Il est inutile de se tracasser, car, si tout est bien prévu, on sait, qu'au jour fixé, l'alevin se nourrira, aussitôt lâché dans son nouveau domaine de chasse. Voici comment nous procédons : Les élèves accomplissent bien tranquillement la résorption presque totale de 
leur vésicule, sans recevoir un atome d'aliment imposé. A l'approche du moment décisif, ils s'agitent puis se mettent à nager comme de vrais poissons. Nous attendons encore quelques jours, et alors seulement, c'est-àdire six à sept semaines après les éclosions - parfois même à l'âge de deux mois, pour les sujets issus des premières pontes - nous commençons à les semer au dehors. Ils sont encore bien vifs, et, le nombre des Truitelles récoltées en automne affermira notre conviction qu'ils n'ont guère souffert de la faim, dans leur prime jeunesse. Disons même que, si les sacs vitellins sont entièrement résorbés, les résultats peuvent encore être excellents : de nombreux exemples nous le prouvent. Toutefois, il est bien entendu que cette remarque n'est pas un conseil.

La condition essentielle qui permet de suivre la marche indiquée, c'est d'employer une eau très froide et quelque peu nourrissante, donc, celle d'un ruisseau, non filtrée, si possible, et apportant sa microfaune et ses matières dissoutes. C'est là un charriage nutritif fort mince, sans dóute, en comparaison du nombre des alevins parqués, et leur résistance prolongée peut nous surprendre. Elle s'explique, nous semble-t-il, par la supposition suivante : les besoins de nourriture extérieure sont probablement très minimes pendant l'achèvement de la résorption, et cela, d'autant plus que la température de l'eau est plus basse. Quoi qu'il en soit, le fait est que, pendant la saison froide, quelques litres par minute de l'eau d'un bon ruisseau, suffisent pour assurer la santé et la vigueur de quelques milliers d'alevins, jusque vers la fin de leur période vitelline.

Quant aux eaux de source, qui présentent en général toutes les qualités requises par le nourrissage, et sont même presque de rigueur dans ce cas, nous les laissons à la Truite arc-en-ciel. Comme l'eau doit être abondante en été, pour la poursuite de l'élevage intensif, et que ces trois circonstances: sources, gros débit, saison sèche, ne se rencontrent pas toujours, il y a là un nouvel écueil de la méthode artificielle.

Prénourrissag.e. - On pratique parfois une sorte de forçage antérieur au traitement naturel. Dans ce cas, les jeunes Truites quittent la salle d'alevinage, vers l'âge de deux à trois mois, ou même plus tard. Ce procédé nous l'avons dit - est rarement utile. C'est un pis aller, justifiable, peutêtre, si l'on ne peut obtenir que l'alevin et son habitat futur soient simultanément prêts pour le déversement ; mais ce pis aller est fondé, en dernière analyse, sur un vieil attachement irraisonné à l'eau de source. Or, l'emploi d'une telle eau, suivi du déversement dans un biotope alimenté par un ruisseau, est une grosse faute quand on veut faire de l'élevage naturel de Truite fario. Si nous envisageons le problème au point de vue des rendements à espérer, nous persistons à préférer la mise en semi-liberté d'alevins " presque résorbés " à celle de sujets stimulés par un "prénourrissage ". On affirme que ceux-ci donnent un meilleur pourcentage de réussite que ceux-1ฉ. Il faut s'entendre: le nombre de truitelles d'un été, fourni par cent fario "élevés au biberon " sera peut-être apparemment supérieur 
à celui que donneront cent autres, plus jeunes et non alimentées, mais en réalité, la balance penchera le plus souvent du côté de ces dernières. Supposons un rendement de 40 à $50 \%$, obtenu en partant d'alevins prénourris. Cela fait un déchet de 50 à $60 \%$, auquel il ne faut pas oublier d'ajouter celưi qui se produit pendant le nourrissage dans les bacs. En d'autres mots, le calcul doit partir du même point dans les deux cas. On constatera alors que l'élimination survenue avant le déversement, rabat fréquemment le pourcéntage vrai, c'est-à-dire le rapport œufs-truitelles à un niveau inférieur au rendement numérique des alevins lâchés plus jeunes et à jeun. Ceci se vérifie encore plus souvent et à plus forte raison, quand il s'agit d'élevage artificiel continu.

Nourrissag.e après coup. - Lorsqu'on manque de place et que l'on désire augmenter la production de truitelles, tout en renonçant aux inconvénients du nourrissage en bacs, l'idée qui vient à l'esprit est de distribuer des aliments artificiels un ou deux mois après le déversement, ce traitement semi-extensif devant permettre de renforcer le nombre des sujets immergés dans les différentes pièces d'eau. Il y a là un calcul rationnel les alevins, étant petits au début, représentent un poids vif minime, que la nature seule nourrira pendant les premières semaines, et, quand celle-cı n'y pourvoira plus suffisamment, à cause de l'accroissement de ce poids, l'alimentation de complément interviendra. En pratique, cette méthode offre des déceptions, car : $-1^{\circ}$ on risque de surpeupler le biotope, pour la période " d'abstinence artificielle " et cela amène des hécatombes; - $2^{\circ}$ l'alevin de fario, ancré dans ses habitudes sauvages, ne vient guère à la nourriture qu'on lui jette ultérieurement. Bref, il est rare qu'on obtienne ainsi une augmentation de rendement.

On pourrait aussi songer à commencer le nourrissage au moment du déversement, mais cela ne nous paraît pas plus recommandable, et pour les mêmes raisons (surpopulation, marchant de pair avec la répugnance envers les aliments imposés), auxquelles nous ajouterons les chances plus grandes d'infection du milieu; car nous supposons l'essai de ce dernier procédé dans des pièces d'eau de dimensions réduites. S'il faut recourir à l'alimentation artificielle, cette éducation spéciale de l'alevin ne peut guère être tentée que dans les bacs, c'est-à-dire sur des masses compactes de jeunes poissons.

Comme c'est précisément cela que nous voúlons éviter, et que les expédients mentionnés, - soit qu'ils visent à améliorer la méthode extensive (intervention avant ou après le déversement), - soit qu'ils tendent à corriger les défauts du traitement intensif (culture et récolte de proies vivantes, nourrissage prématuré) - ne nous satisfont pas davantage, il ne nous reste plus qu'à choisir l'élevage naturel intégral, dont nous pourrons d'ailleurs accroitre la production par des moyens comparables à ceux de l'agriculture. 
Avantages du traitement naturel.

La qualité des produits est un argument invoqué parfois, mais rarement en connaissance de cause, en faveur de la forme d'élevage préconisée ici. Entendons par là, leur valeur comme sujets de repeuplement, lleur pouvoir d'adaptation et de résistance, leur virtualité de croissance. On pourrait rectifier cette assertion, et la ramener dans ses justes limites, en disant que la qualité s'obtient plus facilement par le traitement naturel.

Il saute aux yeux qu'une jeune Truite, qui a passé les 6 ou 8 premiers mois de son existence (période de résorption exceptée) à jouir de grands espaces, à dévorer tout son content de bonnes bouchées vivantes, sans devoir - si nous pouvons risquer cette métaphore - jouer des coudes au milieu de la foule, est un sujet plus " vitaminé " que sa sœur parquée et gavée d'aliment plus ou moins adéquats. Pour celle-ci, cette vigueur ne sera pas atteinte aussi aisément : la résistance aux maladies et à l'asphyxie, lors des transports par exemple, se montrera souvent inférieure.

Nous ne voulons pas dire par là que la truitelle " nourrie " ne convient pas pour les rempoissonnements, comme cela a été affirmé jadis et redit depuis, sous prétexte qu'elle n'est pas aguerrie, qu'elle reste rabougrie, qu'elle devient une proie facile pour ses ennemis, qu'elle succombe danc

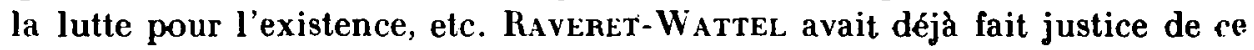
préjugé. L'instinct de la conservation, l'aptitude à rechercher pitance et cachettes ne se perdent pas. Voyons donc moucheronner les milliers de Truitelles, entassées dans les bassins où se pratique le nourrissage intensif, et, concluons pour la faune exogène. Autopsions ces sujets " artificiels ", et nous verrons que la faune endogène égarée dans ces biotopes, plutôt négatifs, est également pillée. Quand à l'instinct de se cacher, regardons comment la jeune fario vient à la nourriture distribuée. Elle sort brusquement de la profóndeur, fonce sur la pâture offerte, et repart comme une flèche, pour savourer, en catimini, le morceau enlevé. Et puis, les Arc-enciel, elles-mêmes, ne montrent-elles pas qu'elles sont taillées pour la lutte, quand on assiste au spectacle de cette ruée qui se déchaîne au moment des repas, et de ces morceaux arrachés à la bouche des concurrentes?

La convenance aux repeuplements est surtout une question de qualilé. Or un trutticulteur soigneux, qui nourrit ses élèves, peut aussi, quoiqu'au prix de plus de peine et d'argent, arriver à produire de la bonne truitelle résistante et viable.

Ce n'est donc pas cela qui nous fait opter résolument pour l'élevage naturel : ce choix, lorsque nous disposons des espaces suffisants, s'appuie sur des motifs plus réels.

1. - Conditions de vie idéales offertes par la nature, à tous les points de viue (bien-être, hygiène, alimentation, etc.) et qu'on est dispensé de remplacer par des expédients. 
2. - Simplicité du traitement extensif et de l'obtention de la qualité par cette voie.

3. - Absence habituelle des troubles d'origine alimentaire et réduction énorme des riqques de maladies infectieuses.

4. - Epargne de main-d'œuvre: la besogne, qui devient saisonnière ne manque, certes pas, au printemps et en automne, mais en été elle se borne à une simple surveillance.

5. - Abolition du souci journalier de la pitance à trouver.

6. - Exigences moins grandes concernant le débit et même la pureté de l'eau.

7. - Utilisation intéressante des pièces d'eau nouvellement créées óloignées. Celles-ci étant plus exposées aux braconniers, il est prudent de les peupler d'un fretin très menu, qui ne donne pas de tentations.

8. - Rendements numériques généralement supérieurs, grâce, notamment, à la suppression quasi totale du déchet dans la population des bacs d'alevinage.

9. - Prix de revient normalement moins élevés. 